\title{
Electronic properties of disordered valence-bond stripes in cuprate superconductors
}

\author{
Matthias Vojta \\ Institut für Theoretische Physik, Universität zu Köln, Zülpicher Straße 77, 50937 Köln, Germany
}

(Dated: Sep 17, 2008)

\begin{abstract}
We calculate single-particle properties of short-range ordered stripe states, using Monte-Carlo simulations of collective charge-density wave (CDW) order parameters coupled to fermions on a $2 \mathrm{~d}$ square lattice. For superconducting bond-centered stripes with a $d$-wave form factor, we find a valence-bond "glass" which coexists with low-energy quasiparticles featuring interference phenomena, in agreement with recent scanning-tunneling-microscopy (STM) measurements on underdoped $\mathrm{Bi}_{2} \mathrm{Sr}_{2} \mathrm{CaCu}_{2} \mathrm{O}_{8+\delta}$ and $\mathrm{Ca}_{2-x} \mathrm{Na}_{x} \mathrm{CuO}_{2} \mathrm{Cl}_{2}$. Together with earlier work, our calculations provide a link between CDW signatures seen in STM and those in magnetic neutron scattering.
\end{abstract}

\section{INTRODUCTION}

Charge-density wave (CDW) phenomena have been detected in a number of superconducting cuprates. Most prominent are the uni-directional spin and charge modulations, termed "stripes", in $\mathrm{La}_{2-x} \mathrm{Ba}_{x} \mathrm{CuO}_{4}$ and $\mathrm{La}_{2-x} \mathrm{Sr}_{x} \mathrm{CuO}_{4}$ (with $\mathrm{Nd}$ or Eu co-doping), being strongest near $1 / 8$ th doping. ${ }^{1-5}$ In other cuprates, notably $\mathrm{Bi}_{2} \mathrm{Sr}_{2} \mathrm{CaCu}_{2} \mathrm{O}_{8+\delta}$ and $\mathrm{Ca}_{2-x} \mathrm{Na}_{x} \mathrm{CuO}_{2} \mathrm{Cl}_{2}$, scanning tunneling microscopy (STM) measurements have found signatures of short-range charge order. ${ }^{6-9}$.

Remarkably, the existence of stripe states was postulated in early theory work on the Hubbard model, ${ }^{10-12}$ far before experimental indications for such phases were found. Later on, ideas of frustrated phase separation as driving force of stripe formation were worked out in detail, ${ }^{13}$ and CDW quantum criticality was proposed as source of both non-Fermi liquid behavior and superconductivity. ${ }^{14}$ For the vast number of subsequent theoretical activities we refer to the review articles Ref. 15,16 .

While the role of charge order for the overall properties of cuprates is under debate, a plausible hypothesis is that tendencies toward charge ordering are common to underdoped cuprates. ${ }^{13-18}$ Even compounds not displaying long-range order are influenced by the proximity to a charge-ordered state. In particular, impurities will act as random-field pinning centers for the collective charge modes, leading to static short-range order (as observed in STM). ${ }^{15,19,20}$ Moreover, charge order will influence the magnetic excitations, believed to be the pairing glue: ${ }^{21}$ It was recently shown ${ }^{22}$ that short-range-ordered stripes give rise to an "hour-glass" magnetic spectrum, very similar to that observed in neutron scattering experiments both on $\mathrm{La}_{2-x} \mathrm{Ba}_{x} \mathrm{CuO}_{4}{ }^{23}$ and $\mathrm{YBa}_{2} \mathrm{Cu}_{3} \mathrm{O}_{6+\delta} .{ }^{24}$

While neutron and X-ray scattering were used to detect superstructure modulations from long-range charge order, ${ }^{1,2,5}$ there is relatively little information on the electronic structure of stripe states. Both STM and photoemission indicate the presence of coherent, gapless nodal quasiparticles (QP) in $(1,1)$ direction, whereas antinodal QP in $(1,0)$ direction are rather incoherent and likely dominated by charge ordering. ${ }^{8,25,26}$ For the compound $\mathrm{La}_{2-x} \mathrm{Ba}_{x} \mathrm{CuO}_{4}$, a $d$-wave-like gap was recently reported ${ }^{27}$ which may be attributed to static stripes or to fluctuating superconductivity. ${ }^{28}$

In this paper, we present a detailed study of local electronic properties of disordered stripe states in cuprates, using a CDW order-parameter approach plus a meanfield theory for the single-particle dynamics. A central ingredient is the $d$-wave-like form factor of the charge order, ${ }^{29}$ which causes the modulations to be located primarily on $\mathrm{Cu}-\mathrm{O}-\mathrm{Cu}$ bonds instead of on $\mathrm{Cu}$ sites. Our results reproduce central features of the STM data of Refs. 9,26. As we employ the same model for the collective CDW modes as in Ref. 22, used there to calculate spin excitations in the presence of disordered stripes, our results provide a link between different probes of stripe physics.

The remainder of the paper is organized as follows: In Sec. II we describe the employed model together with the approximations and their physical background. Sec. III presents the main numerical results, with focus on describing the STM data of Refs. 9,26. A discussion and conclusion closes the paper.

\section{PHENOMENOLOGICAL MODELLING}

Our phenomenological model consists of coupled CDW fluctuations and electrons, with the action $\mathcal{S}=\mathcal{S}_{\psi}+$ $\mathcal{S}_{c}+\mathcal{S}_{c \psi}$. To account for the strong commensuration effects observed experimentally, all fields will be defined for discrete lattice coordinates. ${ }^{30}$

\section{A. Lattice CDW order-parameter theory}

The CDW part $\mathcal{S}_{\psi}$ captures the tendency toward stripe ordering and is identical to that of Ref. 22: Two complex fields $\psi_{x, y}(\vec{r}, \tau)$ represent the amplitude of horizontal and vertical stripe order at wavevectors $\vec{K}_{x, y}$, such that the real field $Q_{x}(\vec{r})=\operatorname{Re} \psi_{x}(\vec{r}) e^{i \vec{K}_{x} \cdot \vec{r}}$ (similarly for $Q_{y}$ ) measures the modulation of both the charge density and bond order (i.e., kinetic energy or pairing amplitude), for $\vec{r}$ on sites and bonds, respectively. Then, $\delta \rho\left(\vec{r}_{j}\right)=Q_{x}+Q_{y}$ is the deviation of the local hole density from its spatial average. We restrict our attention to $\vec{K}_{x}=(\pi / 2,0)$ 
and $\vec{K}_{y}=(0, \pi / 2)$, i.e., a charge modulation period of 4 lattice spacings. ${ }^{6,7}$ The complex phase of $\psi_{x, y}$ represents the sliding degree of freedom of the density wave.

Fluctuations of the charge order are described by a $\psi^{4}$ type theory $\mathcal{S}_{\psi}$ for the $\mathrm{O}(4)$ field $\psi=\left(\psi_{x}, \psi_{y}\right)$. The precise form of $\mathcal{S}_{\psi}$ will determine the character of the fluctuations (amplitude vs. phase). The STM data of Ref. 6, with modulations present everywhere in real space, point toward small amplitude fluctuations; in addition, the calculated spin-fluctuation spectra of Ref. 22 were only compatible with experiment under the assumption of dominant phase fluctuations. Hence, we employ

$$
\begin{aligned}
& \mathcal{S}_{\psi}=\int d \tau \sum_{i}\left[\left|\partial_{\tau} \psi_{i x}\right|^{2}+\left|\partial_{\tau} \psi_{i y}\right|^{2}+s_{x}\left|\psi_{i x}\right|^{2}+s_{y}\left|\psi_{i y}\right|^{2}\right. \\
& +c_{1 x}^{2}\left|\psi_{i x}-\psi_{i+x, x}\right|^{2}+c_{2 x}^{2}\left|\psi_{i x}-\psi_{i+y, x}\right|^{2} \\
& +c_{1 y}^{2}\left|\psi_{i y}-\psi_{i+x, y}\right|^{2}+c_{2 y}^{2}\left|\psi_{i y}-\psi_{i+x, y}\right|^{2} \\
& +u_{1} \psi_{i}^{4}+u_{2} \psi_{i}^{6}+v\left|\psi_{i x}\right|^{2}\left|\psi_{i y}\right|^{2} \\
& \left.+w\left(\psi_{i x}^{4}+\psi_{i x}^{* 4}+\psi_{i y}^{4}+\psi_{i y}^{* 4}\right)\right]
\end{aligned}
$$

with $\psi_{i x} \equiv \psi_{x}\left(\vec{r}_{i}\right)$ and $\psi_{i}^{2} \equiv\left|\psi_{i x}\right|^{2}+\left|\psi_{i y}\right|^{2}$. A combination of $u_{1}<0$ and $u_{2}>0$ suppresses amplitude fluctuations of $\psi$. The quartic $v\left|\psi_{x}\right|^{2}\left|\psi_{y}\right|^{2}$ term regulates the repulsion or attraction between horizontal and vertical stripes; we shall mainly employ $v>0$ leading to stripe-like order (whereas $v<0$ results in checkerboard structures). The phase-sensitive $w$ term provides commensurate pinning and selects bond-centered (instead of site-centered) stripes $^{9,22}$ for $w>0$.

\section{B. Fermions}

To calculate electronic properties in the presence of collective charge modes, we start from a BCS model of fermions on the square lattice of $\mathrm{Cu}$ atoms: ${ }^{31}$

$\mathcal{S}_{c}=\int d \tau \sum_{\vec{k}}\left[\bar{c}_{\vec{k} \sigma}\left(\partial_{\tau}+\epsilon_{\vec{k}}-\mu\right) c_{\vec{k} \sigma}+\Delta_{\vec{k}}\left(c_{\vec{k} \uparrow} c_{-\vec{k} \downarrow}+\right.\right.$ c.c. $\left.)\right]$

where summation over spin indices $\sigma$ is implied. The single-particle dispersion consists of hopping to first, second, and third neighbors, with $t=-0.15 \mathrm{eV}, t^{\prime}=-t / 4$, $t^{\prime \prime}=t / 12$. The chemical potential is $\mu=-0.12 \mathrm{eV}$, leading to a hole doping of $\approx 11 \%$. The pairing is of $d$-wave type, $\Delta_{\vec{k}}=\Delta_{0}\left(\cos k_{x}-\cos k_{y}\right)$ with $\Delta_{0}=24 \mathrm{meV}$.

The coupling to the collective CDW fields $Q_{x, y}$ reads

$$
\begin{aligned}
& \mathcal{S}_{c \psi}=\int d \tau \sum_{i}\left[\kappa_{1} Q_{x}\left(\vec{r}_{i}\right) \bar{c}_{i \sigma} c_{i \sigma}\right. \\
& \quad+\left(\kappa_{2} Q_{x}\left(\vec{r}_{i+x / 2}\right) \bar{c}_{i \sigma} c_{i+x, \sigma}+\kappa_{3} Q_{x}\left(\vec{r}_{i+y / 2}\right) \bar{c}_{i \sigma} c_{i+y, \sigma}\right. \\
& \left.\quad+\kappa_{4} Q_{x}\left(\vec{r}_{i+x / 2}\right) c_{i \uparrow} c_{i+x \downarrow}+\kappa_{5} Q_{x}\left(\vec{r}_{i+y / 2}\right) c_{i \uparrow} c_{i+y \downarrow}+c . c .\right) \\
& \quad+[x \leftrightarrow y]]
\end{aligned}
$$

with $Q_{x}\left(\vec{r}_{i+x / 2}\right)=\left[Q_{x}\left(\vec{r}_{i}\right)+Q_{x}\left(\vec{r}_{i+x}\right)\right] / 2$. The coupling constants $\kappa_{1} \ldots 5$ decide about the electronic struture of the CDW state, by implementing modulations of charge densities and bond kinetic and pairing energies. In the simplest picture, stripes correspond to modulations in the on-site charge densities. Those are induced by $\kappa_{1}$ and lead to a nearly $\vec{k}$-independent ( $s$-wave) CDW form factor $\phi_{2}(\vec{k})=\left\langle c_{\vec{k}+\vec{K}, \sigma}^{\dagger} c_{\vec{k} \sigma}\right\rangle \cdot{ }^{29,31}$ However, local ordering can instead be dominated by physics on $\mathrm{Cu}-\mathrm{O}-\mathrm{Cu}$ bonds: Stripe formation is driven by the competition between kinetic and magnetic energies, both living on bonds. ${ }^{32-34}$ We have recently argued ${ }^{29}$ that such a bond-dominated stripe state will have modulations in $\left\langle c_{i \sigma}^{\dagger} c_{i+\Delta, \sigma}\right\rangle$ with locally different signs on horizontal and vertical bonds, implying a strong $d$-wave component of $\phi_{2}(\mathbf{k})$, see Fig. 1 of Ref. 29. Modulations on bonds are induced by $\kappa_{2 \ldots .}$, with the $d$-wave character encoded, e.g., in $\kappa_{2}=-\kappa_{3}$.

A few remarks are in order: In the advocated model, Eqs. $(1,2,3)$, correlation effects are included via $\epsilon_{\vec{k}}$ being a renormalized quasiparticle dispersion and via $Q_{x, y}$ representing collective CDW tendencies, while genuine Mott physics is absent. Dispersion renormalizations are standard in mean-field theories of correlated electrons; here we refrain from a self-consistent calculation of the dispersion and instead use plausible hopping parameters extracted from photoemission experiments. The separation of degrees of freedom into quasiparticles and collective CDW fields, both with full spatial or momentum dependence, is phenomenological and cannot be rigorously justified. However, e.g., in the context of electrons interacting with antiferromagnetic fluctuations, this has proven to be a fruitful route of investigation. ${ }^{35}$

\section{Observables}

STM experiments determine the spatially resolved local density of states (LDOS), $\rho(\vec{r}, E)$, up to an $\vec{r}$ dependent tunnel matrix element (which depends on the set-point conditions..$^{9,26}$ ) To separate physical modulations from set-point effects, the LDOS ratios

$$
\begin{aligned}
& Z(\vec{r}, E)=\frac{\rho(\vec{r}, E)}{\rho(\vec{r},-E)}, \\
& R(\vec{r}, E)=\frac{\int_{0}^{E} d \omega \rho(\vec{r}, \omega)}{\int_{-E}^{0} d \omega \rho(\vec{r}, \omega)}
\end{aligned}
$$

have been used. In a weakly doped Mott insulator, both $Z$ and $R$ (measuring spectral particle-hole asymmetry) can be shown to be proportional to the hole density. ${ }^{36,37}$ In Ref. 9, spatial modulations were observed in $R(\vec{r}, E)$. In the following, we shall assume that these reflect modulations in the hole density. ${ }^{37,38}$ 

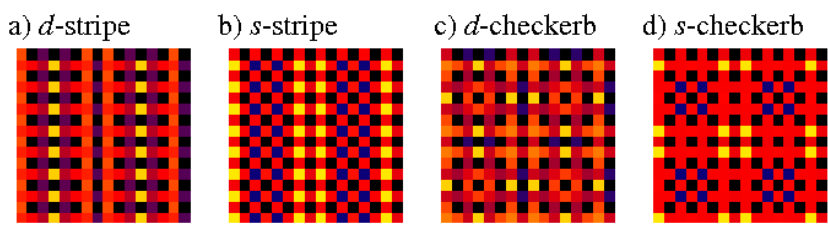

0.06 0.16

FIG. 1: Local densities for ordered CDW states, calculated from $\mathcal{S}_{c}+\mathcal{S}_{c \psi}$ for constant $\psi$. The figures show both site and bond densities, $\left\langle 1-c_{i \sigma}^{\dagger} c_{i \sigma}\right\rangle$ and $\left\langle c_{i \sigma}^{\dagger} c_{i+\Delta, \sigma}+\text { h.c. }\right\rangle^{40}$ for $8^{2} \mathrm{Cu}$ sites; black squares are centers of $\mathrm{CuO}_{2}$ plaquettes. a) Stripes with $\kappa_{1}=0, \kappa_{2}=-\kappa_{3}=0.03,-\kappa_{4}=\kappa_{5}=0.007$ ( $d$-wave). b) Stripes with $\kappa_{1}=0.022, \kappa_{2} \ldots 5=0$ (s-wave). c) Checkerboard $[d$-wave as in a)]. d) Checkerboard [ $s$-wave as in b)].

\section{Perfect CDW order}

Perfectly ordered CDW states are described by $\mathcal{S}_{c}+$ $\mathcal{S}_{c \psi}$, with $\psi_{x, y}$ taken to be constant. From the diagonalized fermionic Hamiltonian all electronic properties can be obtained.

Sample results for the real-space densities of different types of CDW are displayed in Fig. 1. Here, $\psi_{x}=$ $(1+i) / \sqrt{2}, \psi_{y}=0$ for the bond-centered stripes in panels a) and b), while $\psi_{x}=\psi_{y}=(1+i) / \sqrt{2}$ for the checkerboards in panels c) and d). The couplings $\kappa$ were taken to induce $s$-wave-like [panels b) and d)] or $d$-wave-like [panels a) and c)] modulations, and the overall $\kappa$ amplitude was chosen such that the resulting modulation of fermionic densities is about $30-40 \%$. To facilitate comparison with STM data, ${ }^{9}$ which show a strong modulation on the bonds of the $\mathrm{CuO}_{2}$ plane, ${ }^{39}$ we included the bond charge densities $\left\langle c_{i \sigma}^{\dagger} c_{i+\Delta, \sigma}+\right.$ h.c. $\rangle$ (i.e. kinetic energies) in Fig. 1 - those are shown in between the squarelattice sites. ${ }^{40}$ Clearly, Fig. 1a with $d$-wave stripes is most compatible with experiment. ${ }^{9}$

\section{E. Pinning and adiabatic approximation}

The treatment of $\mathcal{S}_{c}+\mathcal{S}_{\psi}+\mathcal{S}_{c \psi}$ requires additional input. Pinning is important especially in the disordered phase of $\mathcal{S}_{\psi}$ : Quenched disorder (e.g. from dopant impurities) acts as a random field and renders static a shortrange ordered stripe configuration. In such a situation, the electronic properties can be approximately calculated by diagonalizing $\mathcal{S}_{c}+\mathcal{S}_{c \psi}$ for fixed static configurations of $\psi_{x, y}$. We generate these from classical lattice Monte Carlo (MC) simulations of $\mathcal{S}_{\psi}$, using a standard Metropolis algorithm at a finite effective temperature $(T=1)$ in a regime where the stripe correlation length is of order $\xi \approx 10 .^{41}$

The numerical procedure parallels that of the adiabatic approximation of Ref. 22, with the difference that the ingredient of pinning is crucial to obtain a static signal in STM. (The presence or absence of pinning was irrelevant to the finite-frequency spin fluctuations described in Ref. 22.) In contrast to earlier work dealing with fermionic properties in the presence of disordered stripes, ${ }^{42}$ our modelling implements the $d$-wave bond character, and it properly describes short-range order via $S_{\psi}(1)$, i.e., stripe segments coexist with checkerboard domain walls. ${ }^{19,20,22}$

\section{F. Choice of parameters and validity of approximation}

The parameters of $\mathcal{S}_{c}+\mathcal{S}_{c \psi}$, Eqs. $(2,3)$, used in our simulations are taken as in the static-stripe calculation above, i.e., for the fermionic sector we use values for $t$, $t^{\prime}, t^{\prime \prime}$, and $\Delta$, which are standard in the BCS mean-field description of cuprates, and the couplings $\kappa$ are taken as in Fig. 1a.

The CDW part of the action, $\mathcal{S}_{\psi}(1)$, is designed to capture the complicated non-universal physics of the strongly correlated CDW formation on the lattice scale. The combination of $s_{x, y}, u_{1}$ and $u_{2}$ decides about the importance of amplitude vs. phase fluctuations of the $\mathrm{CDW},{ }^{22}$ we have used $s_{x}=s_{y}=-4 \ldots-3, u_{1}=-1.15$, $u_{2}=0.1$. Choosing $v=0.2$ prefers stripes over checkerboards, but allows for some checkerboard structure between stripe domains. ${ }^{22}$ Finally, $w=0.05$ is taken for a moderate commensurate lattice pinning toward bondcentered stripes. The precise values of the mass $s$ and the gradient $c$ were used to tune the CDW correlation length $\xi$, which was between 10 and 30 in our simulations. Note that an overall scale factor in $\mathcal{S}_{\psi}$ is free and determines the typical amplitude of $\psi$ which we have normalized to unity. The $\mathcal{S}_{\psi}$ parameters here are identical to those used in Figs. 1a, 2a of Ref. 22 for the description of the spin excitations of fluctuating stripes. Moreover, the charge configurations generated from the MC simulations visually match the STM results in the sense that short and medium stripe segments coexist with checkerboard-like domain walls. This property is robust with respect to parameter changes of $20 \%$ and more, provided that the correlation length $\xi$ is kept fixed. As we employ classical MC simulations for $\mathcal{S}_{\psi}$, with time gradients absent, the parameters cannot be translated directly into physical energies or velocities.

Our approach assumes that a mean-field picture of both superconductivity and charge order is a reasonable starting point for the description of cuprates. The adiabatic approximation neglects inelastic processes and stripe dynamics, which can be justified if the latter is slow (compared to the observed fermions), as happens in the proximity to a CDW ordering transition. Thus, the approximation is invalid for energies below a typical stripe fluctuation frequency; for strong impurity pinning this scale is small or zero. ${ }^{41}$ The quasiparticle picture in cuprates may break down at elevated energies; as far as this happens due to inelastic physics, it is not captured by our approach (while some elastic disorder physics is 


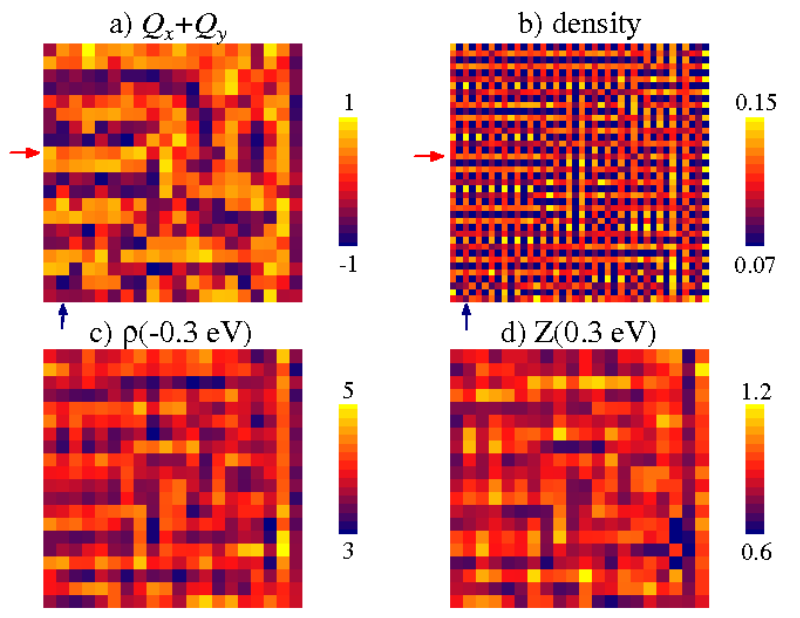

FIG. 2: Local observables for pinned short-range ordered stripes ( $\kappa$ parameters as in Fig. 1a, correlation length $\xi \approx 10$ ), showing $20^{2} \mathrm{Cu}$ sites of the $64^{2}$ sample. a) Order parameter field $Q_{x}+Q_{y}$. b) Local density (including bonds) as in Fig. 1. c) LDOS at $-0.3 \mathrm{eV}$. d) $Z(\vec{r}, E)$ at $0.3 \mathrm{eV}$. The arrows mark the rows/columns along which the LDOS is shown in Fig. 5.

captured).

Further, we assume that dimerization and bond order are the driving forces behind stripe ordering, ${ }^{22,34}$ whereas magnetic long-range order is less important. For simplicity, we therefore neglect both order and fluctuations in the triplet channel. Note that this does not mean that we ignore local-moment physics entirely, but instead we assume that those moments form singlet valence bonds, which is accounted for by modulated hoppings $\left(\kappa_{2,3}\right)$ in $\mathcal{S}_{c \psi}$ (3). We note that the coupling to magnetic fluctuations will contribute to the broadening primarily of antinodal quasiparticles, ${ }^{43}$ but a calculation including spatial disorder and inelastic processes is beyond the scope of the present paper.

\section{NUMERICAL RESULTS}

We now turn to the numerical results obtained from $\mathcal{S}_{c}+\mathcal{S}_{\psi}+\mathcal{S}_{c \psi}$ for short-range ordered stripes.

\section{A. Local densities}

Fig. 2 displays the order parameter field $\left(Q_{x}+Q_{y}\right)$ together with the resulting fermionic charge density, the LDOS $\rho(\vec{r}, E)$, and $Z(\vec{r}, E)$ at a high energy of $0.3 \mathrm{eV}$, for one fixed $\psi_{x, y}$ configuration ${ }^{41}$ for a $d$-wave coupling in $\mathcal{S}_{c \psi}$. The stripe modulation, being prominent on the bonds, leads to a large contrast in Fig. 2b, while the contrast in both the site-LDOS $\rho$ and $Z$ (Figs. 2c,d) is weaker. (Both $\rho$ and $Z$ show strong modulations around the gap energy, Fig. 5 below.) The result in Fig. $2 \mathrm{~b}$ has a striking similarity to the "glassy" structures in
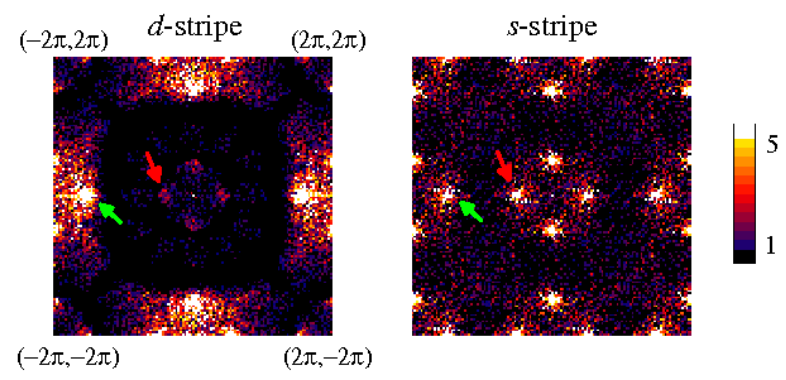

FIG. 3: Fourier-transformed local density (from sites and bonds ${ }^{40}$ ) for short-range ordered stripes, obtained from averaging $40 \mathrm{MC}$ configurations on a $64^{2}$ lattice. Left: $\kappa_{1}=0$, $\kappa_{2}=-\kappa_{3}=0.03,-\kappa_{4}=\kappa_{5}=0.007$ ( $d$-wave). Right: $\kappa_{1}=0.022$, $\kappa_{2 \ldots 5}=0$ ( $s$-wave). The dark/light (red/green) arrows indicate the peaks at $(\pi / 2,0)$ and $(3 \pi / 2,0)$. (The strong peaks at the zone boundary arise from artificially setting the density to zero in the plaquette centers.)

Figs. 3,4 of Ref. 9. In particular, the modulation locally breaks the $\mathrm{C}_{4}$ rotation symmetry down to $\mathrm{C}_{2}$ and is primarily located on the $\mathrm{Cu}-\mathrm{O}-\mathrm{Cu}$ bonds. The latter fact - which originates in the $d$-wave form factor - can be nicely seen in the Fourier-transformed density, Fig. 3. Stripe order is manifest in peaks at $(\pi / 2,0),(0, \pi / 2)$ and $(3 \pi / 2,0),(0,3 \pi / 2)$, with the signal at $(3 \pi / 2,0)$ being much stronger compared to $(\pi / 2,0)$ (whereas for $s$-wave stripes the peaks are roughly equal in intensity). Again, this is in agreement with STM data, Fig. 6 of Ref. 9.

We note that the present comparison between theory and experiment does not easily allow to deduce the amplitude of the actual modulations: The only observables free of set-point effects are $Z$ and $R$. However, a reliable calculation of these has to has to cope with Mott physics not included in our model. ${ }^{38}$ A rough estimate, however, relates the experimentally observed $R$ contrast of $\approx \pm 30 \%$ to a bond modulation of similar magnitude.

\section{B. Nodal quasiparticles and quasiparticle interference}

One outstanding feature of the STM results on underdoped $\mathrm{Ca}_{2-x} \mathrm{Na}_{x} \mathrm{CuO}_{2} \mathrm{Cl}_{2}$ is the presence of quasiparticle interference (QPI) features in the low-energy spectra, in a situation where the high-energy spectra are dominated by period- 4 modulations. ${ }^{26}$

Our calculations qualitatively reproduce this physics. For QPI to occur, we have to add realistic disorder as source of QP scattering: Following Ref. 44, we use a combination of $2 \%$ extended potential scatterers (strength 40 meV, size 1.2), $5 \%$ extended pairing scatterers (strength $\Delta_{0}$, size 1.5 ), and $0.2 \%$ pointlike unitary scatterers (strength $2.5 \mathrm{eV}$ ). The real-space results of such a calculation are displayed in Fig. 4. Both the LDOS and the $Z$ map at higher energies are clearly dominated by stripe segments, whereas the signal below $\approx 25 \mathrm{meV}$ shows the typical QPI modulations (compare e.g. Fig. 3c of 

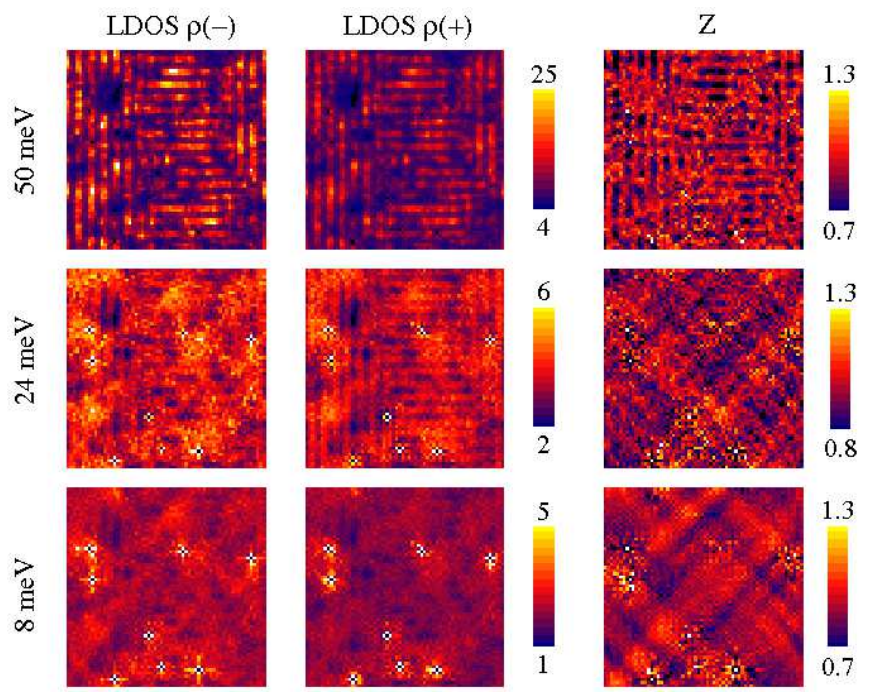

FIG. 4: LDOS $\rho(\vec{r}, E)$ at negative (left) and positive (middle) bias, together with $Z(\vec{r}, E)$ (right), for pinned short-range ordered stripes with additional impurities on a $64^{2}$ lattice, at energies 50, 24, $8 \mathrm{meV}$ (from top to bottom). $\kappa$ parameters are as in Fig. 1a, for details see text.

Ref. 26). Extracting the scattering wavevectors from the Fourier transform of our data (not shown) is difficult due to the small system size; the only unambigous peak is at the so-called $q_{7}$ wavevector (corresponding to the diagonal modulations in $Z$ at low $E$, Fig. 4).

We point out two features of our results. (i) The $Z$ map is more sensitive to QPI than the LDOS, because, to leading order, QPI modulations at positive and negative $E$ are anti-phase, while stripe modulations are inphase. Nevertheless, the strong period-4 modulations seen in the experimental low-energy $\operatorname{LDOS}^{26}$ are likely due to set-point effects. (ii) Real-space localization of antinodal QP cannot be made responsible for the loss of QPI at higher $E$. We have calculated the inverse participation ratio (not shown) as an indicator of localization, and have observed no localization signatures on scales up to several $\xi$ (while these length scales are sufficient to observe QPI).

More generally, the compatibility of stripes with longlived nodal QP has been pointed out in the past. ${ }^{29,34,45-47}$ For small stripe amplitude, this already follows from the fact that the ordering wavevector $\mathbf{Q}$ does not connect the nodal points. ${ }^{34,46}$ In our case, nodal QP survive even for large stripe amplitude due to the $d$-wave character of the charge order (provided that the $s$-wave component remains small). ${ }^{29}$

Within our simulations, the survival of coherent nodal QP in the presence of disordered stripes is also seen in the LDOS spectra in Fig. 5, taken along two different line cuts indicated in Fig. 2. While strong inhomogeneities occur at elevated energies, in particular near the gap energy (note the period-4 modulation in Fig. 5b around $-50 \mathrm{meV}$ ), the low-energy part of the LDOS is essentially homogeneous ${ }^{47}$ again in striking similarity to STM data. $^{8,9}$ (A detailed comparison of our spectra with experiment reveals several differences, which we believe to be related to Mott physics not captured here.)

\section{CONCLUSIONS}

We have determined electronic properties of shortrange ordered stripe states, coexisting with superconductivity. Agreement with salient features of STM experiments, in particular stripy LDOS modulations at elevated energies coexisting with QP interference at low energies, is found for valence-bond stripes with $d$-wave-like form factor, singling out a specific mean-field plus stripe disorder model.

As the same collective-mode description was used earlier to model magnetic excitations in the presence of fluctuating or disordered stripes, our calculations give a unified account of stripe signatures seen in STM and in neutron scattering, and strongly indicate that similar physics underlies the modulated states observed in different underdoped cuprates.

Very recent STM experiments ${ }^{48}$ indicate the quasiparticle interference disappears not only at high energies, but at a very specific location in momentum space, approximately at the boundary of the antiferromagnetic Brillouin zone. Such a feature is not part of the present theory, and likely requires to take into account either antiferromagnetic fluctuations or other precursors of strong Mott physics.

\section{Acknowledgments}

We thank J. C. Davis, H. Takagi, and A. Yazdani for discussions, and R. K. Kaul, S. Sachdev, T. Vojta, and A. Wollny for collaborations on related work. This research was supported by the DFG through SFB 608 (Köln) and the Research Unit FG 538.
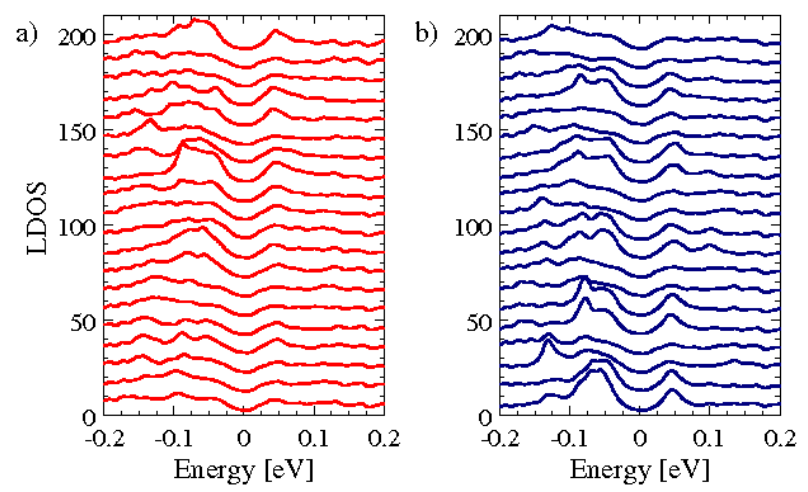

FIG. 5: LDOS spectra corresponding to the data in Fig. 2, along the two paths indicated by arrows in Figs. 2a,b. Spectra have been broadened by $7 \mathrm{meV}$ and shifted for clarity. 
1 J. M. Tranquada, B. J. Sternlieb, J. D. Axe, Y. Nakamura, and S. Uchida, Nature 375, 561 (1995).

2 J. M. Tranquada, J. D. Axe, N. Ichikawa, A. R. Moodenbaugh, Y. Nakamura, and S. Uchida, Phys. Rev. Lett. 78, 338 (1997); J. M. Tranquada, J. Phys. Chem. Solids 59, 2150 (1998).

${ }^{3}$ K. Yamada, C. H. Lee, K. Kurahashi, J. Wada, S. Wakimoto, S. Ueki, H. Kimura, Y. Endoh, S. Hosoya, G. Shirane, R. J. Birgeneau, M. Greven, M. A. Kastner, and Y. J. Kim, Phys. Rev. B 57, 6165 (1998).

${ }^{4}$ V. J. Emery, S. A. Kivelson, and J. M. Tranquada, Proc. Natl. Acad. Sci. USA 96, 8814 (1999).

5 P. Abbamonte, A. Rusydi, S. Smadici, G. D. Gu, G. A. Sawatzky, and D. L. Feng, Nat. Phys. 1, 155 (2005).

6 T. Hanaguri, C. Lupien, Y. Kohsaka, D.-H. Lee, M. Azuma, M. Takano, H. Takagi, and J. C. Davis, Nature 430, 1001 (2004).

7 M. Vershinin, S. Misra, S. Ono, Y. Abe, Y. Ando, and A. Yazdani, Science 303, 1995 (2004).

8 K. McElroy, D.-H. Lee, J. E. Hoffman, K. M. Lang, J. Lee, E. W. Hudson, H. Eisaki, S. Uchida, and J. C. Davis, Phys. Rev. Lett. 94, 197005 (2005).

9 Y. Kohsaka, C. Taylor, K. Fujita, A. Schmidt, C. Lupien, T. Hanaguri, M. Azuma, M. Takano, H. Eisaki, H. Takagi, S. Uchida, and J. C. Davis, Science 315, 1380 (2007).

10 J. Zaanen and O. Gunnarsson, Phys. Rev. B 40, 7391 (1989).

11 H. Schulz, J. de Physique 50, 2833 (1989).

12 K. Machida, Physica C 158, 192 (1989); M. Kato, K. Machida, H. Nakanishi, M. Fujita, J. Phys. Soc. Jpn 59, 1047 (1990).

13 V. J. Emery and S. A. Kivelson, Physica C 209, 597 (1993), ibid. 235-240, 189 (1994).

14 C. Castellani, C. Di Castro, and M. Grilli, Phys. Rev. Lett. 75, 4650 (1995); J. Phys. Chem. Solids 59, 1694 (1998).

15 S. A. Kivelson, I. P. Bindloss, E. Fradkin, V. Oganesyan, J. M. Tranquada, A. Kapitulnik, and C. Howald, Rev. Mod. Phys. 75, 1201 (2003).

16 A. H. Castro Neto and C. Morais Smith, in: Strong Interactions in Low Dimensions, D. Baeriswyl and L. Degiorgi, eds. (Kluwer, 2004), pg. 277.

17 J. Zaanen, Physica C 317, 217 (1999).

18 D. J. Scalapino and S. R. White, Found. Phys. 31, 27 (2001).

19 A. Del Maestro, B. Rosenow, and S. Sachdev, Phys. Rev. B 74, 024520 (2006).

20 J. A. Robertson, S. A. Kivelson, E. Fradkin, A. C. Fang, and A. Kapitulnik, Phys. Rev. B 74, 134507 (2006).

21 J. Zaanen et al. Nature Phys. 2, 138 (2006).

22 M. Vojta, T. Vojta, and R. K. Kaul, Phys. Rev. Lett. 97, 097001 (2006).

23 J. M. Tranquada, H. Woo, T. G. Perring, H. Goka, G. D. Gu, G. Xu, M. Fujita, and K. Yamada, Nature 429, 534 (2004).

24 S. M. Hayden, H. A. Mook, P. Dai, T. G. Perring, and F. Dogan, Nature 429, 531 (2004).

${ }^{25}$ K. M. Shen, F. Ronning, D. H. Lu, F. Baumberger, N. J. C. Ingle, W. S. Lee, W. Meevasana, Y. Kohsaka, M. Azuma, M. Takano, H. Takagi, and Z.-X. Shen, Science 307, 901 (2005).

26 T. Hanaguri, Y. Kohsaka, J. C. Davis, C. Lupien, I. Yamada, M. Azuma, M. Takano, K. Ohishi, M. Ono, and H.
Takagi, Nature Phys. 3, 865 (2007).

27 T. Valla, A. V. Fedorov, J. Lee, J. C. Davis, and G. D. Gu, Science 314, 1914 (2006).

28 Q. Li, M. Huecker, G. D. Gu, A. M. Tsvelik, J. M. Tranquada, Phys. Rev. Lett. 99, 067001 (2007).

29 M. Vojta and O. Rösch, Phys. Rev. B 77, 094504 (2008).

30 Note that the present stripe order-parameter theory explicitly takes into account lattice effects, which goes beyond standard Landau or Landau-Ginzburg approaches [e.g., O. Zachar, S. A. Kivelson, and V. J. Emery, Phys. Rev. B 57, 1422 (1998)].

31 We use the standard fermionic notation where $c^{\dagger}, c$ are canonical operators, whereas $\bar{c}, c$ denote the corresponding Grassmann numbers in the coherent-state path integral.

32 S. Sachdev, Rev. Mod. Phys. 75, 913 (2003).

33 S. Sachdev and N. Read, Int. J. Mod. Phys. B 5, 219 (1991).

34 M. Vojta and S. Sachdev, Phys. Rev. Lett. 83, 3916 (1999); M. Vojta, Y. Zhang, and S. Sachdev, Phys. Rev. B 62, 6721 (2000).

35 A. V. Chubukov, D. Pines, and J. Schmalian, in: The Physics of Conventional and Unconventional Superconductors, K.H. Bennemann and J. B. Ketterson, eds. (Springer, 2002).

36 P. W. Anderson and N. P. Ong, J. Phys. Chem. Solids 67, 1 (2006).

37 M. Randeria, R. Sensarma, N. Trivedi, and F.-C. Zhang, Phys. Rev. Lett. 95, 137001 (2005).

38 In our calculations, the quantity $Z$ is sensitive to stripe modulations and QPI, but does not measure "Mottness" due to the mean-field character of $S_{\psi}+S_{c \psi}$.

39 Microscopics beyond one-band models may be required to obtain a full picture of bond order in cuprates.

40 The bond densities $\left\langle c_{i \sigma}^{\dagger} c_{i+\Delta, \sigma}+\right.$ h.c. $\rangle$ have been re-scaled with a fixed numerical factor such that they equal the site hole densities $\left\langle 1-c_{i \sigma}^{\dagger} c_{i \sigma}\right\rangle$ in the homogeneous superconducting reference state. This prescription appears appropriate for a weakly doped Mott insulator and ensures that both highly doped sites (implying stronger hopping) and strong bonds lead to a similar contrast.

41 The real-space results in this paper have been calculated from a single $\mathrm{MC}$ configuration of $\mathcal{S}_{\psi}$ each. We have also performed simulations of $\mathcal{S}_{\psi}$ with different pinning potentials included (whose microscopic form, however, is not known). The obtained results are similar to the ones shown here (provided that $\xi$ is comparable).

42 See e.g.: M. I. Salkola, V. J. Emery, and S. A. Kivelson, Phys. Rev. Lett. 77, 155 (1996); G. Seibold, F. Becca, F. Bucci, C. Castellani, C. Di Castro, and M. Grilli, Eur. Phys. J. B 13, 87 (2000).

43 S. Sachdev, M. Troyer, and M. Vojta, Phys. Rev. Lett. 86, 2617 (2001).

44 T. S. Nunner, W. Chen, B. M. Andersen, A. Melikyan, and P. J. Hirschfeld, Phys. Rev. B 73, 104511 (2006).

45 M. Granath, V. Oganesyan, S. A. Kivelson, E. Fradkin, and V. J. Emery, Phys. Rev. Lett. 87, 167011 (2001).

46 E. Berg, C.-C. Chen, and S. A. Kivelson, Phys. Rev. Lett. 100, 027003 (2008).

47 M. Granath, Phys. Rev. B 77, 165128 (2008).

48 Y. Kohsaka et al., Nature 454, 1072 (2008). 